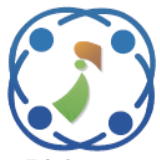

\title{
Effective Maintenance of Path Stability Using ACO-PLF Mechanism in MANETs
}

\author{
Bellam Purushotham ${ }^{1 *}$ \\ Choda Durga Venkata Subba Rao ${ }^{1}$ \\ ${ }^{I}$ Department of Computer Science and Engineering, \\ Sri Venkateswara University College of Engineering, Sri Venkateswara University, Tirupati, India \\ * Corresponding author’s Email: purush_bmp@yahoo.com
}

\begin{abstract}
Ad hoc networks are the infrastructure-less networks that are easily deployable and self-configuring, with each constituent node performing like a transceiver. The nodes are free to move subjectively in Mobile ad-hoc networks (MANET). Thus, the network's wireless topology may be haphazard and may alter rapidly. The main important aspect to be handled is choosing a proper path for transmitting data in case of Link Failure (LF). The main concern of the research work is maintaining the path stability in the occurrence of LF in the network. Ant Colony Optimization (ACO) is used for optimizing the Routing Protocols (RP) such as Ad-hoc on demand Distance Vector (AODV). The Prediction of LF (PLF) mechanism is used for choosing the stable/proper path in the occurrence of node LF in the maintenance phase of distance vector RP based on Neighbour Node (NN). Hence, AODV-ACO-PLF is used for efficient transmission of data packets in the ad-hoc networks by maintaining stable path in-case of LF. The path stability is also maintained in presence of LF in the networks by choosing a different path using PLF Prediction Mechanism. The "AODV-ACO-PLF" methodology is used for finding the ideal way to communicate with the source and destination by giving better results in End-to end delay (6\%), drop (5\%), packet delivery ratio (7\%), and energy consumption (7.5\%) than existing methodology such as Path Selection Algorithm based on Hamming Distance and delay parameters (PSA-HD) and Predict Failure in AODV (PF-AODV) methodology in MANET.
\end{abstract}

Keywords: Ad-hoc on demand distance vector, Ant colony optimization, Maintenance phase, Mobile ad-hoc networks, Neighbour node and prediction of link failure.

\section{Introduction}

Mobile Ad-Hoc Network (MANETs) is one kind of self-configuring and dynamic wireless network, which processes several movable user equipment. The router utilizes the connection state steering worldview and floods their data to every single other course in the network. Every single portable node in the system independently refreshes their data in the connection state [1]. Multi cast AODV (MAODV) depends on the multicast RP by finding stable route and adjust to network topology changes [2]. A productive routing algorithm should be steady and furthermore energy saving also adjustable in dynamic network condition. [3]. High versatility of the MANET nodes diminishes the dependability of network communication. The high versatility of the nodes makes it extremely hard to predict the dynamic routing topology in a dynamic system [4]. Connection breakage starts the procedure of rerouting either at the sender node or at the source node [5]. Privacy-preserving and truthful detection of packet dropping attacks in Manet uses HLA based public-auditing architecture by ensuring packet-loss notification by every individual node in the networks. The detection of misbehaving nodes in the network, while transmitting data from source to destination is not addressed in a proper way [6].

The Ad-hoc On Demand Multiple Path Distance Vector (AOMDV) protocol is the most appropriate for detecting multiple paths to the destination. The identification of mobility of mobile nodes can be tackled by retransmission strategy. The AOMDV protocol does not provide better results in case of throughput and control packet overhead [7]. The multipath choice is done by utilizing three unique 
schemes called short-multipath, stable-multipath and short-stable multipath. Stable Multipath RP (SMRP) uses a link predication model to predict the link break among the multiple paths [8]. Efficient ant-colony optimization algorithm for routing in MANETs in which destination-node is chosen and trades the packets (specialists) between the source and the destination. The algorithms are needed for adopting larger dimensional topology of MANET by addressing security to the data packets [9]. ACO is a population based autocatalytic system of finding the best ideal arrangements as per the regular activities of insect. The pheromone updating parameters can be further enhanced [10]. A node protection protocol allows for the establishment of stable connections in MANETs. The time variants locations and velocities affect the communication reliability under increasingly realistic conditions of node movement and occasional failure [11]

To overcome this problem, "AODV-ACO-PLF" is implemented for increasing the parameters such as, End-to end delay, drop, packet delivery ratio, energy consumption. The PLF prediction mechanism is used for choosing proper path in presence of LF in networks. The RP used as AODV routing and route is optimized by enhanced ACO technique. Thus "AODV-ACO-PLF" Methodology provides a stable path by predicting the link breakage is used based on neighbour node distance and time taken to receive the data from sender node to receiver nodes. Thus, the prediction algorithm in maintenance phase provides better results in term of End-to end delay, drop, packet delivery ratio, and energy consumption in adhoc networks than PSA-HD and PF-AODV methodology in MANET.

The rest of this paper is organized as follows: Section 2, reports on related work. Section 3, presents a review on "AODV-ACO-PLF" Methodology in MANETs. Section 4, demonstrated the simulation parameters of the "AODV-ACO-PLF" and the conclusion of this work is made in Section 5.

\section{Related work}

D. Garg, and P. Gohil [12] introduced a novel routing algorithm for MANETS based on the swarm intelligence. In this method, for optimal path selection, ACO algorithm was used. The maintenance route has to be done periodically, with the selection of optimal path for data transmission in the network. But the optimal path section transmit data to the destination is not more effective for larger networks.

G. Singh, N. Kumar, and A.K. Verma [13] presented an Innovative ANTALG by contributing an irregular arrangement of source and destination node issues. Hence, those issues are rectified by using Ant (specialists) techniques between irregular nodes in networks. Here this calculation and execution parameters were related with the AODV, Ant Dynamic Source Routing (ADSR), and Hybrid Ant Colony Optimization Routing algorithm (HOPNET), presume that the proposed calculation gives better throughput and reduced average end to end delay, packet drop, average jitter. Major limitation is the movement of packets and Ant's agent through the network doesn't changes the routing policy with the less security in networks.

T. Senthilkumaran, and V. Sankaranarayanan [14] implemented a method for dynamic congestiondetection and control routing (DCDR) in ad-hoc networks based on the assessments of the average queue-length at the node level. The DCDR method performance decrease in-case of packet losses with error-induced packets is major drawbacks.

R. Vadivel, and V.M. Bhaskaran [15] has presented adaptive, reliable and congestion-control $\mathrm{RP}$ to solve the congestion and route errors using route assortment in MANETs. The node sends signal to its neighbours utilizing the congestion indication bit. A node ascertains many elective ways and disseminates the bypass traffic over various paths in the networks. This scheme introduces a very less number of nodes by varying the rate control values in the simulation with on-the-spot information collection of the networks. The scheme address only QoS parameter compared with existing methodologies.

A.M. Desai, and R.H. Jhaveri [16] implemented to secure routing ad-hoc network predictive method to diminish sequence number attacks, which discovers mischievous nodes during route discovery. The network variants not enhanced by incorporating novel secure routing solutions, energy conservation, authentication and key distribution techniques, which is the major drawbacks in networks.

Calduwel Newton Pitchai [17] introduced Path selection algorithm based on Hamming distance (PSA-HD) to enhance the link stability to develop a model for path selection by determining a stable path to reach the destination. The identification of position of node failure in the path carried out by selecting an alternate link from the failure node. The Minimum number of hamming distance and minimum delay provides better results in term of mobility in the network. Major limitation is that link expiration time does not survive for a longer duration of time with minimum connected neighbours. 


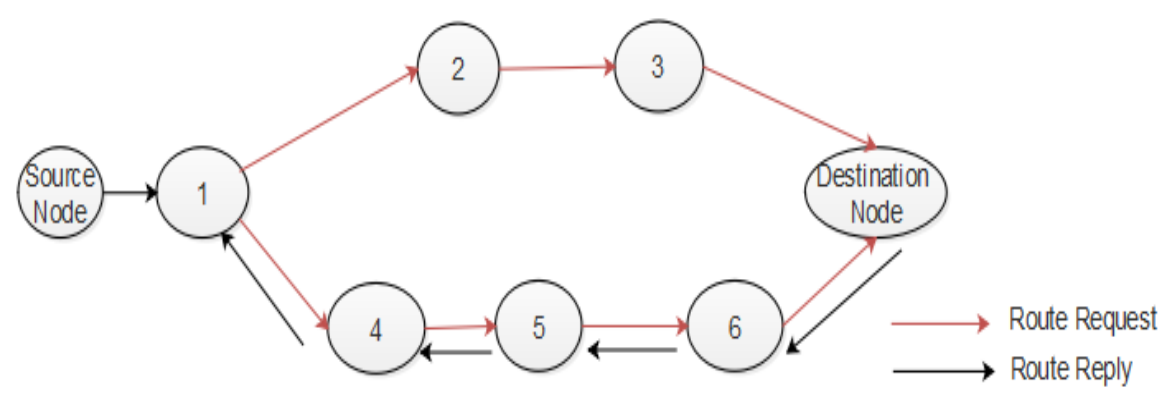

Figure. 1 AODV RPs-data transfer

S. Maamar, and B. Abderezzak [18] implemented a prediction of link failure in AODV protocol (PFAODV) to provide quality of service in MANET. The main aim is to add a mechanism to predict link failure based on signal strength by making more robust to anticipate on link breaking methods. Lack of node initiates in this phase earlier than other to promote nodes near the destination in larger networks.

\section{3. "AODV-ACO-PLF based on NN" methodology}

The "AODV-ACO- PLF based on NN" algorithm is used to maintain stable path by selecting proper route in terms of node LF in ad-hoc networks by providing better results in end-to-end delay, drop, packet delivery ratio, and energy consumption. The link recovery plays very important role in ad-hoc networks. AODV RP is used for routing in the MANET. Though AODV is a reactive RP, routes resolute only when needed. The message type of the AODV is represented as different types such as, Route Requests (RREQs), Route replies (RREPs) and Route error (RERRs). Based on the testing and analysing AODV, the "AODV-ACO-PLF" methodology to predict the LF using delay time while transmitting data packets from source to destinations. The brief explanation of "AODV-ACO-PLF" is addressed below;

\subsection{AODV-RP}

The AODV RP deals with two functions such as Route Discovery and Route Maintenance. To find the new route, the Route Discovery work is selected, and the link failure is fixed by using an existing route by Route Maintenance function. The receptive convention does not keep a permanent route table. AODV is quickly able to analyse the changes in network topology. The Data transfer of AODV RPs is given in Fig. 1.

\subsection{ACO technique}

ACO is used in ad-hoc networks for optimizing the RP on the bases of ant-nature. The ACO is adapted for optimizing difficult functions, which is capable of imitating the ability of ants to process knowledge. The key concepts are the potential solutions to be accelerated toward better or more optimum solutions. The formulating path stability problems are also known as an optimization problem that directs the network to its optimum point in the network. The PLF prediction algorithm is used to uphold the path stability in secured path, when there is a LF in the transmission path. The probability for ant $l$ at node $\mathrm{k}$ moving to node $l$ at generation $u$.

$$
Q_{j, i}^{k}(u)=\frac{\tau_{j, k}(u) d_{j, k}^{-\beta}}{\sum_{u \in \Gamma_{i}^{k}} \tau_{j, v} d_{j, v}^{-\beta}} k \in \Gamma_{j}^{k}
$$

Where $\tau_{j, k}$ is the intensity of the pheromone on edge $\tau_{j, k}, d_{j, k}$ is the distance between nodes $i$ and $k$, $\Gamma_{u}^{k}$ is the set of nodes to be visited by ant $k$ position at node $I$ to make the solution feasible and $\beta<0$ is given in Eq. (1).

Once all the ants have built their tours, the pheromone is updated on all edges $j \rightarrow k$ agreeing to a global pheromone updating rule, which is mentioned in the Eq. (2), (3) \& (4).

$$
\tau_{j, k}(t+1)=(1-\rho)_{j, k}(t)+\Delta \tau_{j, k}(t)
$$

Where

$$
\begin{aligned}
& \Delta \tau_{j, k}(t)=\sum_{k=1}^{N_{p}} \Delta \tau_{j, k}^{k}(t) \\
& \Delta \tau_{j, k}^{k}(t)=\left\{\begin{array}{c}
\frac{Q}{L_{k}}, \quad \text { if }(j, k) \in \text { tourdonebyant } \\
0, \text { otherwise }
\end{array}\right.
\end{aligned}
$$

$(1-\beta)$ is the pheromone decay parameter $(0<$ 


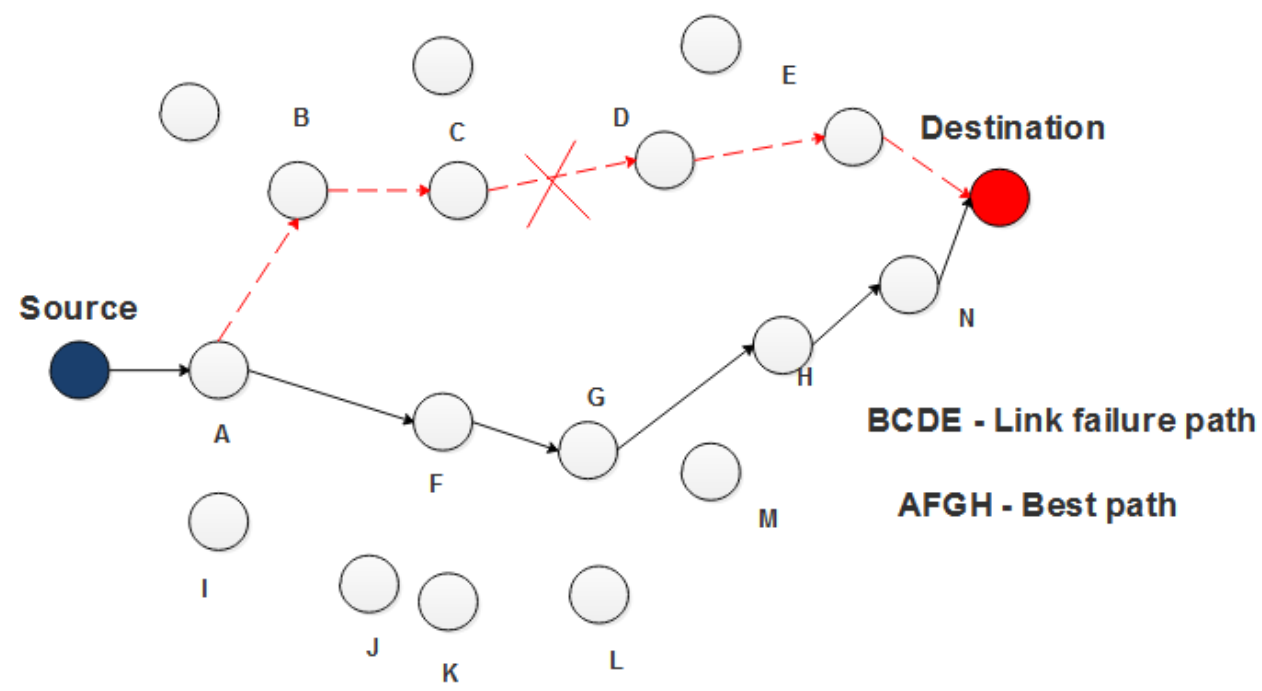

Figure. 2 Basic diagram of choosing different path in case of LF

$\rho<1$ ) where it represents the track evaporation when the ant chosen a source destination and decide to move $L_{k}$ is the length of the tour accomplished by ant $k$ and $\mathrm{m}$ is the no. of ants.

\subsection{PLF based prediction on neighbor node in presence of $\mathbf{L F}$}

MANET must be able to establish reliable communication channels which are protected by failure recovery protocols. This research work is centered on securing the route of mobile especially on mobile ad-hoc communication within the sight of LF to improve by finding effective stable communication channels. Fig. 2 shows, basic diagram of choosing different in case of LF

The Data transmission between sources to destination takes place. If there is any presence of LF in the path, it chooses another path in the network. In the Figure, (B-C-D-E) path the link is not secured to choose, so it chooses another path such as, A-F-G-M$\mathrm{H}-\mathrm{N}$.

\subsubsection{PLF in maintenance phase}

AODV protocol is based on the distance vector algorithm, where path between two nodes is evaluated when needed (i.e. when a node wants to send data packets it initiates discovery phase for finding a new path to different phases such as during the transfer phase and during maintenance phase). A set of control packets, such as RREQ, RREP, a Route Error (RERR), RRepAck (Route Reply Acknowledgement) and Hello messages are used in the discovery and maintenance process. A routingtable is connected to each node to store information as Dest address, active neighbour lists, hops number to reach the destination. PLF mechanism uses the knowledge of HELLO message. In the network communication range, the concept of "HELLO message" are sent by all nodes in the networks via. Neighbouring nodes. When nodes receive a number of HELLO messages through Link, which is considered to be good state and can be used for routing of packets. If there is any lack of HELLO messages in the link, it is said to be LF which occurs in the networks. Hence, PLF algorithm detects the time delay occurring between neighbour nodes. As in AODV, it depends on sending Hello message to neighbouring nodes by choosing dynamic route at standard intervals and with extreme short distance. A break at a route does not affect the rest of the routes and therefore the use of another route is always possible to transmit data.

\subsubsection{Estimation of time delay based on neighbor nodes}

In unique condition, a continuous disconnection causes considerable packet loss because of absence of interchange ways (routes) and the remaking of new route produce an extra volume packets (directing) control. PLF is mainly depends upon the no. of sensor-node existent and mobility node with its distance. In network, it's important to calculate any disconnection probable on active route based on distance and time. The time delay values inform us about the link quality and it depends on node mobility (I.e. distance). The calculation of mobility node distance is from its initial position and delay-time duration of HELLO message. PLF algorithm indicates about the connection quality improvement (increasingly steady) and diminishes of packet loss (probability failure) in the routing table. The PLF is used to make link management more robust and also 
on connection failure for improving QoS parameters in the networks.

PLF mainly depends upon the no. of sensor in the transmission -path and the distance between the sensor nodes. The least distance selection with various number of sensors in the networks is given in Eq. (5).

$$
\tau D=\tau 1+\tau 2+\tau 3+\ldots \tau n+\cdots
$$

Where, $\tau$ is a uniform time delays for ad-hoc sensor node pairs $(1,2)(2,3),(3,1)$ and $(n, n+1)$ respectively. Circular topology keeps the nodes at equidistance. Therefore, node pair delay $\tau 1+\tau 2+$ $\tau 3$ will be equal. Let $\tau$ be the uniform time delay for all sensor node pairs in delay time, which is given in Eq. 6.

$$
\tau=\tau 1=\tau 2=\tau 3 \ldots .+\tau n \ldots+. .
$$

This is the minimum DT time of a time duration in WSNs. When distance of the neighbour's node is in remote area, a discovery of path of the destination node occurs between different hops to rescue the transmission time in the network for transmitting data. Fig. 3, shows nodes with $z 1, z 2$ and $z i$ are in close distance with each other and neighbors to $Z s$ on one side and $\mathrm{Zd}$ the other. $S$ is the source and $D$ is the destination.

When node $Z i$ moves away due to mobility, its signal weakens (tends towards zero) and disconnection probability increases.

Two factors are addressed below.

i. $Z i$ Moves towards $X d$ and leave the range of $Z s$ (break of segment $[Z s, Z i]$ ), then $Z s$ tries to rebuild road pieces $[Z s, Z p, Z i, Z d]$, where $p$ is one of neighbour at one hop from $Z s$, which is shown in Fig. 4.

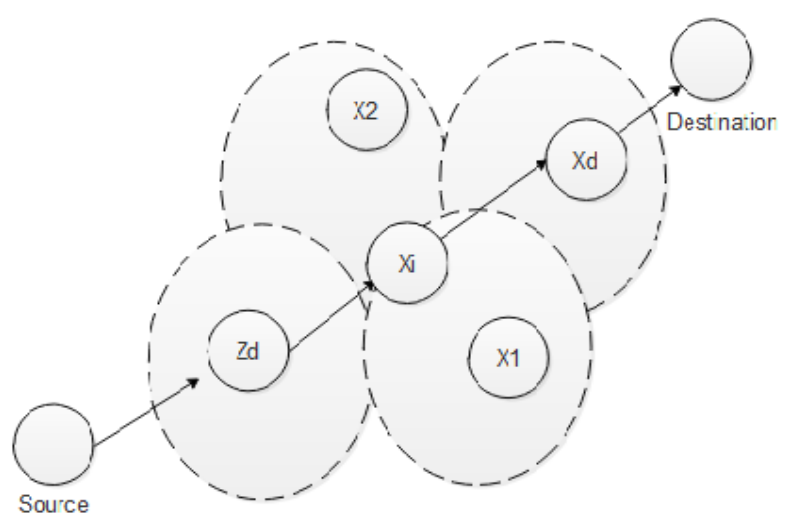

Figure. 3 Neighbour node distance placement

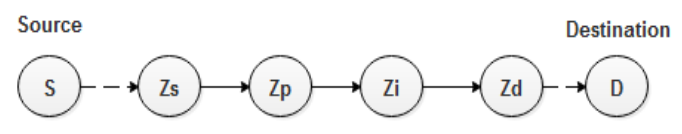

Figure. 4 Reconstructing in $Z s$ side

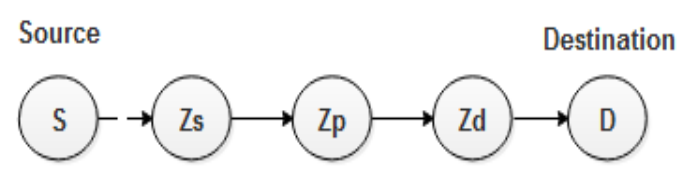

Figure. 5 Reconstructing in xd side

ii. $\quad Z i$ moves towards $X s$ and leave the range of $Z d$ (break of the segment $[Z i, x d]$, then $Z s$ tries to rebuild road pieces $[Z s, Z p, Z d]$, where $p$ is one of the neighbor at one hop from $Z s$, which is shown in Fig. 5.

Therefore, the "AODV-ACO-PLF based on NN" is used for finding optimal path to communicate with the source and destination by providing better results in End-to-end delay, drop, packet delivery ratio, and energy consumption.

The Fig. 6, Shows the flow chart of the overall routing process. Here a finite number of mobile nodes is organized in the specified area and initially, the source and destination are assigned. Once source and destination are defined, then source node broadcast an RREQ to all the neighbour nodes. The route establishment method is done using AODV RP. The Route Reply Packet (RREP) with source request is sent to the neighbour nodes, if the dropping of packets in the network is identified in the networks. After receiving the packet, the source will put the responding node to its failure list. Once it is put on the failure list by announcing to the networks, then knowledge based learning has been applied for confirmation of the distance and speed of the neighbouring nodes. Almost all node in the network get information about the presence of LF.

\section{Simulation results}

The Simulation is defined as the process toward structuring a model of a real-time system and leading with this model for understanding the behaviour of the system as well as assessing different procedures for the operations of the network. The "AODV-ACOPLF based Neighbor nodes" for maintaining the stable path in presence of LF using NS-2, which provide better results in End-to end delay (6\%), drop $(5 \%)$, packet delivery ratio (7\%), and energy 


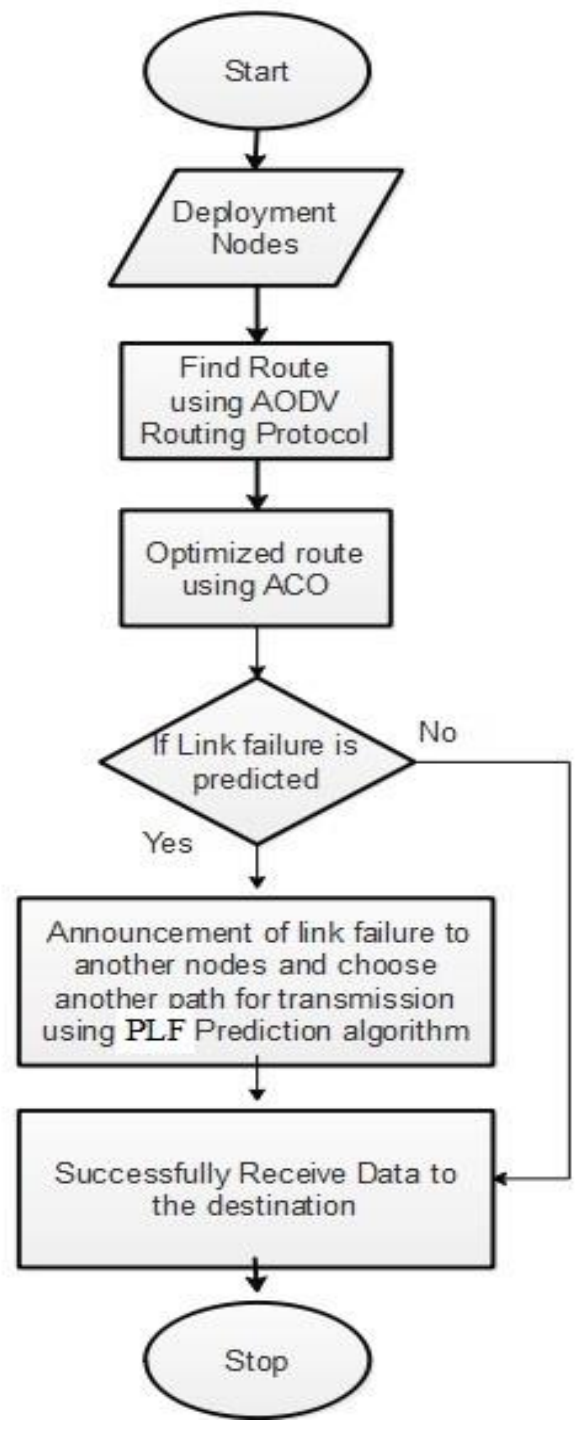

Figure. 6 Flow chart of the overall AODV-ACO-PLF methodology

consumption (7.5\%) compared with PSA-HD [17] and PF-AODV [18]. In which PSA-HD is based on hamming distance to enhance the link stability to choose best path in networks. PF-AODV provide quality of service using signal strength to predict another segment before break occurs.

(i) End-to-End Delay: Difference between Sending time of packets and receiving time of packets is known as delay. End-to-end delay denotes to the time-taken for a packet to be communicated across a network from source-to-destination, given in Eq. (7).

$$
d_{\text {end-end }}=n\left[d_{\text {trans }}+d_{\text {prop }}+d_{\text {queue }}\right]
$$

Where,

$d_{\text {end-end }}=$ end - to - end delay

$d_{\text {trans }}=$ transimission delay $d_{\text {prop }}=$ propagation delay

$d_{\text {proc }}=$ processing delay

$d_{\text {queue }}=$ Queuing delay

$n=$ number of links (Number of routers -1$)$

(ii) Packet drop/Packet loss: Difference between the total amounts of packets sends by source node to the packet received to the destination node is known as the packet drop/packet loss, which is given in Eq. (8).

$$
\text { Drop }=\frac{\text { Total no.of packet send-Packetreceived }}{\text { Total number of simulation }}
$$

(iii) Packet Delivery Ratio (PDR): Based on a total number of packets received in a ratio by a total number of destination packet sent by the source node, given in Eq. (9).

$$
\text { Packet Delivery Ratio }=\frac{(\text { Received Packet })}{\text { Sent packets }}(100)
$$

(iv) Energy consumption: The huge number of nodes are equivalent to the huge amount of received energy consumption. A node drops a particular amount of energy for every packet transmission and reception, which is given in Eq. (10).

$$
\text { Energy }=\frac{\text { amount of energy for every packets }}{\text { total simulation time }}
$$

\subsection{Simulation Topology}

The Comparison analysis of "AODV-ACO-PLF" with existing "PSA-HD" [17] with "PF-AODV" [18] is evaluated by varying the random node. Tables 1 and 2 illustrate the "AODV-ACO-PLF", "PSA-HD" and "PF-AODV" of various parameters such as endto end delay, drop, packet delivery ratio, and energy consumption.

Comparison analysis of AODV-ACO-PLF is evaluated by varying the simulation time. The Fig. 7, 8,9 , and 10 , shows the comparison of the delay, drop, packet delivery ratio and energy Consumption between PSA-HD and PF-AODV.

The Comparison of time vs. delay between AODV ACO-PLF with PSA-HD and PF-AODV is plotted in Fig. 7. The delay value decreased $6 \%$ in AODVACO-PLF method, when compared with the PSAHD and PF-AODV by differentiating simulation times in Secs. Red line indicates proposed method. 
Table 1. Comparison between AODV-ACO-PLF with PSA-HD [17] and PF-AODV [18]

\begin{tabular}{|c|c|c|c|c|c|c|}
\hline \multirow{2}{*}{$\begin{array}{c}\text { Simulation Time } \\
(\mathbf{m} / \mathbf{s})\end{array}$} & \multicolumn{3}{|c|}{ Delay } & \multicolumn{3}{|c|}{ Del Ratio } \\
\cline { 2 - 7 } & PF-AODV & PSA-HD & $\begin{array}{c}\text { AODV- } \\
\text { ACO-PLF }\end{array}$ & PF-AODV & PSA-HD & $\begin{array}{c}\text { AODV-ACO- } \\
\text { PLF }\end{array}$ \\
\hline 7.02 & 1.365 & 1.480 & 1.234 & 23.467 & 23.345 & 23.897 \\
\hline 12.03 & 1.472 & 1.380 & 1.346 & 23.578 & 23.479 & 23.680 \\
\hline 17.05 & 1.593 & 1.634 & 1.456 & 24.778 & 24.672 & 24.897 \\
\hline 22.06 & 1.609 & 1.690 & 1.567 & 24.898 & 24.755 & 24.989 \\
\hline 23.08 & 2.797 & 2.889 & 1.678 & 25.767 & 25.644 & 25.996 \\
\hline
\end{tabular}

Table 2. Comparison between AODV-ACO-PLF with PSA-HD [17] and PF-AODV [18]

\begin{tabular}{|c|c|c|c|c|c|c|}
\hline \multirow{2}{*}{$\begin{array}{c}\text { Simulation Time } \\
(\mathbf{m} / \mathbf{s})\end{array}$} & \multicolumn{3}{|c|}{ Drop } & \multicolumn{3}{|c|}{ Energy } \\
\cline { 2 - 7 } & PF-AODV & PSA-HD & $\begin{array}{c}\text { AODV- } \\
\text { ACO-PLF }\end{array}$ & PF-AODV & PSA-HD & $\begin{array}{c}\text { AODV-ACO- } \\
\text { PLF }\end{array}$ \\
\hline 7.02 & 134 & 139 & 128 & 24.199 & 24.337 & 24.152 \\
\hline 12.03 & 147 & 160 & 136 & 25.219 & 25.452 & 25.168 \\
\hline 17.05 & 156 & 167 & 142 & 26.278 & 26.565 & 26.173 \\
\hline 22.06 & 176 & 186 & 156 & 27.234 & 27.753 & 27.158 \\
\hline 23.08 & 180 & 187 & 178 & 28.678 & 28.893 & 28.516 \\
\hline
\end{tabular}

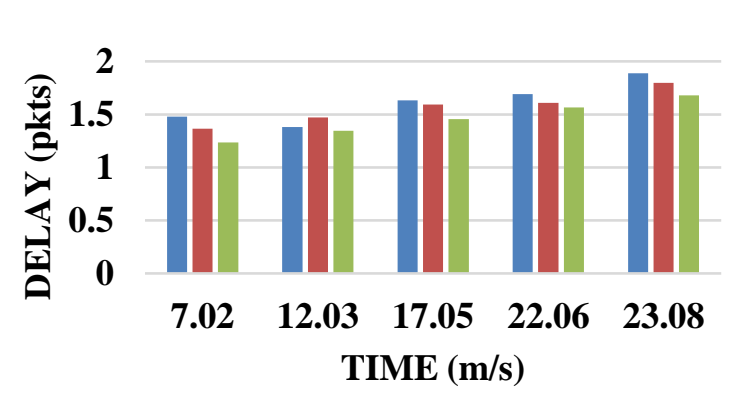

$\square$ PSA-HD $\square$ PF-AODV $\backsim$ AODV-ACO-PLF

Figure.7 Time vs. delay

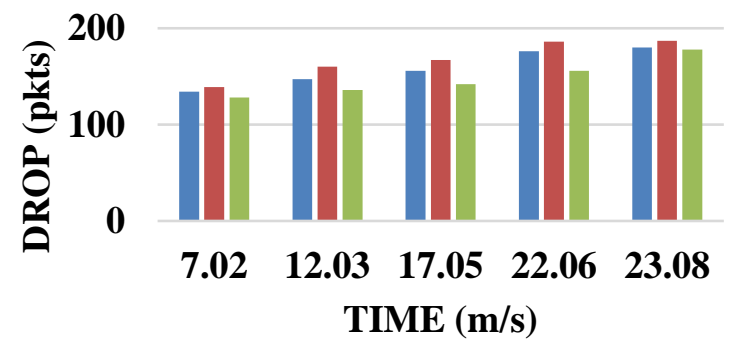

$\square$ PF-AODV $\square$ PSA-HD $\square$ AODV-ACO-PLF

Figure. 8 Time vs. drop

The Comparison of time vs. drop between AODV-ACO-PLF with PSA-HD and PF-AODV is plotted in Fig. 8. The drop value decreased (5\%) in AODV-ACO-PLF method, when compared with the PSA-HD and PF-AODV by differentiating simulation time in Secs. Red line indicates proposed method.

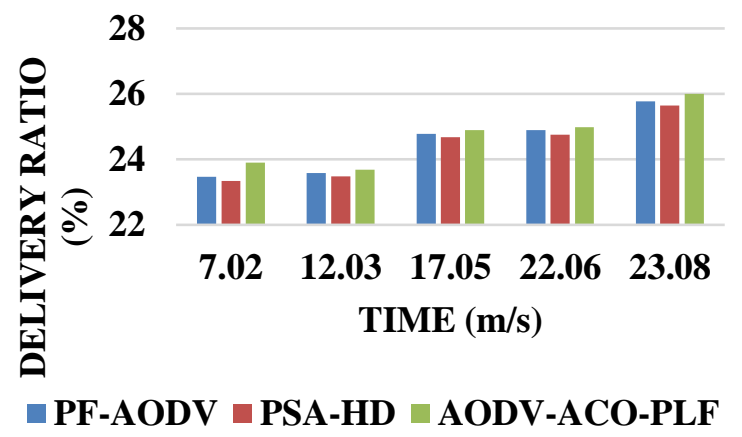

Figure. 9 Time vs. delivery ratio

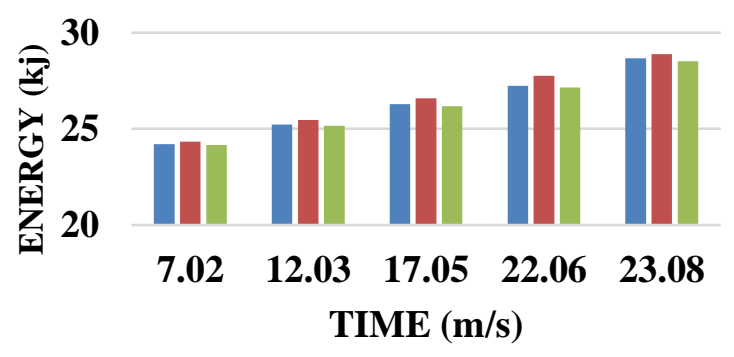

øPF-AODV $\approx$ PSA-HD $\approx$ AODV-ACO-PLF

Figure. 10 Time vs. energy consumption

The Comparison of time vs. delivery ratio between AODV-ACO-PLF with PSA-HD and PFAODV is plotted in Fig. 9. The delivery ratio value increased $(7 \%)$ in AODV-ACO-PLF method, when compared with the PSA-HD and PF-AODV by differentiating simulation time in Secs. Red line indicates proposed method.

The Comparison of time vs. energy between 
Table 3. Simulation parameters

\begin{tabular}{|l|l|}
\hline \multicolumn{2}{|c|}{ Parameters used for Traffic Model } \\
\hline Simulation Time & 50 Seconds \\
\hline Type of traffic & UDP/CBR \\
\hline Packets Size & 500 \\
\hline Packet rate & 500 \\
\hline Area Size & 500 X 500 m \\
\hline \multicolumn{2}{|c|}{ Parameters used for physical and link layers } \\
\hline MAC Protocol & IEEE 802.11 \\
\hline Propagation Model & Two-ray Ground \\
\hline Antenna Model & Omni-antenna \\
\hline Network Interface Type & Wireless (Ad-hoc) \\
\hline \multicolumn{2}{|c|}{ Parameters used for Mobility Model } \\
\hline $\begin{array}{l}\text { Number of Wireless } \\
\text { Nodes }\end{array}$ & Random deployment of \\
\hline Mobility Model & Rodes \\
\hline \multicolumn{2}{|c|}{ Random Waypoint } \\
\hline Methodology Employed & AODV-ACO-PLF \\
\hline Simulation used & NS2 \\
\hline
\end{tabular}

AODV-ACO-PLF with PSA-HD and PF-AODV is plotted in Fig. 10. The energy consumption value decreased (7.5\%) in AODV-ACO-PLF method, when compared with the PSA-HD and PF-AODV by differentiating simulation time in Secs. Red line indicates proposed method.

\subsection{Simulation setup}

The Sensor nodes are randomly distributed in the area. The random number of Nodes are distributed randomly in which each and every packet starts its journey from a random location to a random destination with randomly chosen speed. The mobility and traffic generation are defined with the type of antenna defined. The two-ray ground radiopropagation model is used. The source-destination pairs are spread randomly over the network. In traffic model used are UDP/CBR traffic sources are used. The Source-destination pairs are spread randomly over the network. The simulation parameters are given in Table 3.

\section{Conclusion}

In AODV-ACO based PLF prediction Mechanism is used for transmitting data from source to destination in presence of LF in the ad-hoc networks. The path stability is maintained using a PLF mechanism in presence of LF by selecting a different path in the network. The PLF is done in the AODV RP in the maintenance phase. The AODVACO-PLF mechanism modified route maintenance, when the primary route facing failure. Besides dropping data packets as original AODV, the modification route maintenance scheme uses backup route decided on basis of route reliability for forwarding data packets towards the destination. Hence, the AODV-ACO-PLF is used for avoiding unstable path in the network and finding proper route for transmitting data from source to destination by providing better results in End-to end delay (6\%), drop (5), packet delivery ratio (7\%), energy consumption $(7.5 \%)$ than PSA-HD and PF-AODV techniques. As the future work, the notification node is explicated in the network for choosing the optimal path in occurrence of link failure.

\section{References}

[1] P. Rajeswari and T.N. Ravi, "A Novel SERIeS Algorithm for Enhancing Link Stability and Security in Manet", Journal of Applied Security Research, Vol.12, No.2, pp.287-303, 2017.

[2] H. Xia, S. Xia, J. Yu, Z. Jia, and E.H.M. Sha, "Applying link stability estimation mechanism to multicast routing in MANETs", Journal of Systems Architecture, Vol.60, No.5, pp.467-480, 2014.

[3] S. Hao, H. Zhang, and M. Song, "A Stable and Energy-Efficient Routing Algorithm Based on Learning Automata Theory for MANET", Journal of Communications and Information Networks, Vol.3, No.2, pp.52-66, 2018.

[4] G. Singal, V. Laxmi, M.S. Gaur, and V. Rao, "Moralism: mobility prediction with link stability based multicast RP in MANETs", Wireless Networks, Vol.23, No.3, pp.663-679, 2017.

[5] G. Singal, V. Laxmi, V. Rao, S. Todi, and M.S. Gaur, "Improved multicast routing in MANETs using link stability and route stability", International Journal of Communication Systems, Vol.30, No.11, pp.e3243, 2017.

[6] T. Shu and M. Krunz, "Privacy-preserving and truthful detection of packet dropping attacks in wireless ad hoc networks", IEEE Transactions on mobile computing, Vol.14, No.4, pp.813-828, 2015.

[7] V.D. Savane and V.G. Kasabegoudar, "Path Stability Mechanism in Mobility based AOMDV for MANETS", International Journal of Computer Applications, Vol.76, No.3, 2013.

[8] I. Alagiri, V. Madhuviswanatham, and P. VenkataKrishna, "Efficient Data Transfer by Mobility Adjustment Algorithm for Clustered Mobile Ad-Hoc Networks", Cybernetics and Information Technologies, Vol.14, No. 2, pp.5064, 2014. 
[9] P.K. Kumar and V.R. Prasad, "Efficient Ant Colony Optimization (ACO) based Routing Algorithm for MANETs", Global Journal of Computer Science and Technology, Vol.15, No.3, 2015.

[10] P. Sachar and V. Khullar, "Genetic Algorithm Using MapReduce-A Critical Review. imanager's", Journal on Cloud Computing, Vol.2, No.4, pp.35, 2015.

[11] A. Zadin and T. Fevens, "Maintaining path stability with node failure in mobile ad hoc networks", Procedia Computer Science, Vol.19, pp.1068-1073, 2013.

[12] D. Garg, and P. Gohil, "Ant Colony Optimized Routing for Mobile Ad Hoc Networks (MANET)", International Journal of Smart Sensors and Ad Hoc Networks, Vol.2, No.3, 2012.

[13] G. Singh, N. Kumar, and A.K. Verma, "Antalg: An innovative aco based routing algorithm for manets", Journal of Network and Computer Applications, Vol.45, pp.151-167, 2014.

[14] T. Senthilkumaran and V. Sankaranarayanan, "Dynamic congestion detection and control routing in ad hoc networks", Journal of King Saud University-Computer and Information Sciences, Vol.25, No.1, pp.25-34, 2013.

[15] R. Vadivel and V.M. Bhaskaran, "Adaptive reliable and congestion control RP for MANET", Wireless Networks, Vol.23, No.3, pp.819-829, 2017.

[16] A.M. Desai and R.H. Jhaveri, "Secure routing in mobile Ad hoc networks: a predictive approach", International Journal of Information Technology, pp.1-12, 2018.

[17] C.N. Pitchai and N.R. Robert, "PSA-HD: Path Selection Algorithm based on Hamming Distance to Enhance the Link Stability in Mobile Ad-hoc Networks", International Journal of Intelligent Engineering and Systems, Vol.11, No.1, pp.259-266, 2018.

[18] S. Maamar and B. Abderezzak, "Predict Link Failure in AODV Protocol to provide Quality of Service in MANET", IJ Computer Network and Information Security, Vol.3, pp.1-9, 2016. 